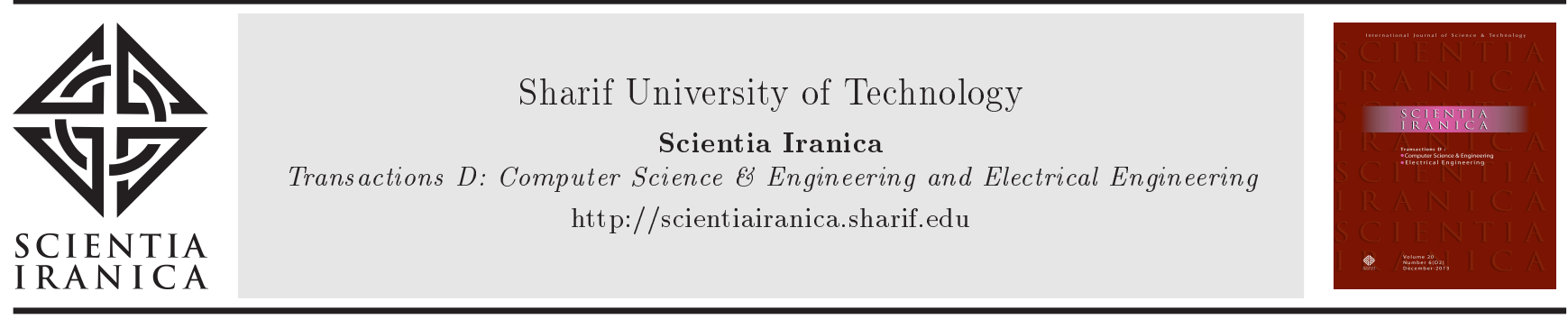

\title{
Using grey wolf optimizer to minimize voltage total harmonic distortion of a salient-pole synchronous generator
}

\author{
D. Perin ${ }^{\mathrm{a}}$, A.D. Karaoglan ${ }^{\mathrm{b}, *}$, and K. Yilmaz ${ }^{\mathrm{a}}$ \\ a. ISBIR Electric Company, Department of R \& D, 10150, Balikesir, Turkey. \\ b. Department of Industrial Engineering, Balikesir University, 10145, Balikesir, Turkey. \\ Received 31 January 2021; received in revised form 13 May 2021; accepted 2 August 2021
}

\section{KEYWORDS}

Maxwell simulation;

Nature inspired

algorithms;

Design optimization;

Electric machines.

\begin{abstract}
It is important to evaluate optimum design parameters of Synchronous Generator (SG) to obtain the desired Total Harmonic Distortion (THD). This study aims to determine the optimum rotor design parameters of SG using Grey Wolf Optimizer (GWO) algorithm. To this end, regression modeling is performed to mathematically model the relationship of the selected rotor design parameters (slot pitch, center slot pitch, and damper width) with THD (response). These factors have not been previously investigated in the related literature. Next, optimization is applied to this regression equation using GWO. Maxwell simulations are used in conjunction with numerical experiments. The GWO results are compared with the results of Genetic Algorithm (GA). The results indicate that the GWO algorithm can be well adapted to similar optimization processes and effectively used. As a result, the voltage THD of the SG is reduced to 0.3951 under acceptable magnetic flux conditions. This GWO-aided optimization study is significant in that it demonstrates how SG performance can be improved by making minor changes to the production line as part of mass production without changing the outer diameter and dimensions of SG.
\end{abstract}

(C) 2023 Sharif University of Technology. All rights reserved.

\section{Introduction}

Total Harmonic Distortion (THD) is the quality of voltage or current. A harmonic is the sinusoidal component of a periodic wave or quantity whose frequency is an integral multiple of the fundamental frequency. The continuous deformation of the waveform, voltage, or current waveform can be separated into different

*. Corresponding author. Tel.: +902666121194

E-mail addresses: tsr16@isbirelektrik.com.tr (D. Perin);

deniz@balikesir.edu.tr (A.D. Karaoglan);

tsr14@isbirelektrik.com.tr (K.Yilmaz) amplitudes and phases with more sinus waveforms, while the frequency of these components is the multiple of the basic frequency. The shape of the output voltage is a sinusoidal wave under normal conditions. This means that THD is zero (or close to zero). When the output signal gets distorted due to various harmonic distortion components, the effective distortion due to all the individual components are considered together. THD represents a percent value and is calculated by taking into account the percentage of the total harmonic components and their fundamental components. Harmonics have always been a problem with industrial loads. As THD increases, the probability of damaging the electrical machine increases. Therefore, minimizing 
the THD in electric machine design is essential to maintain its quality and sustainability [1-3].

Alternator design parameters such as slot pitch, center slot pitch, damper width, slot geometry, slot opening width, alternator diameter, and similar alternator design parameters affect THD. In Synchronous Generator (SG), back electromagnetic force (back EMF) generated in the air gap between the rotor and the stator distorts the sinusoidal wave in synchronous alternator voltage. Fluctuations in voltage cause increase in THD value. The back EMF formed in the air gap between the stator and the rotor is damped with the help of damper windings, and the sine wave form of the voltage is maintained. In this study, no changes have been made to the stator structure (slot geometry, slot opening width, etc.) and size (inner diameter, outer diameter, etc.) in SG, which we have optimized. The changes in the outer diameter of this mass-produced product will affect the dimensions of the components in the generator group where the alternator will be assembled and will require a production line alteration from the beginning to the end. Therefore, in this study, the effect of the position of the damper windings in the rotor on the THD value in a synchronous alternator was investigated. Determining the correct position of the damper windings was considered the most cost-effective and effective way to make proper design changes for the manufacturer. Given its critical role in the positioning of damper windings, slot pitch, center slot pitch, and damper width values were taken as the basis and an attempt was made to obtain minimum THD in this way.

Many metaheuristic algorithms, especially nature-inspired ones, can be found in the literature and are quite popular. In particular, swarm intelligence is very popular among these approximation methods. These techniques have the capability to optimize problems successfully. Optimization of magnetic device parameters to reduce the THD was investigated in many studies. However, few relevant research studies that are limited have already used metaheuristics (especially on swarm intelligence). Metaheuristic studies focus on the use of Genetic Algorithm (GA) [4-10], Particle Swarm Optimization (PSO) [11-15], Clonal Selection Algorithm (CSA) [16], Ant-Lion Optimizer (ALO) [17], and Adaptive Mosquito Blood Search algorithm (AMBS) [18]. There are also remarkable studies that have discussed the effect of damper winding on the SG [19-26].

This study investigates salient-pole SG design optimization to minimize voltage THD. The remarkable studies recently presented on the proposed subject are given as follows: Jiji et al. [27] used virtual prototyping with the help of Finite Element Analysis (FEA) to optimize the design parameters of the third harmonic excitation system for a low-voltage salient-pole SG and then, they managed to reduce the THD. Nuzzo et al. [28] studied the design optimization of a 4-MVA salient-pole $\mathrm{SG}$ to reduce the damper cage loss and improve the no-load voltage THD. De la Cruz et al. [29] dealt with design optimization of a $5 \mathrm{~kW}$ Permanent Magnet SG (PMSG) to reduce the voltage THD. Sparga et al. [30] studied the design optimization for the salient-pole rotor of an SG using FEA to improve the THD. Wang et al. [31] investigated the design optimization for a $400 \mathrm{kVA}$ salient-pole SG using FEA and improved the voltage THD. Choi et al. [32] evaluated slot design optimization for an SG in a bulb turbine to improve the power and voltage THD and then, used FEA to calculate the magnetic flux distribution. Dai et al. [33] used GA and Taguchi in conjunction to optimize the design of a Surface-Mounted PM Synchronous Machines (SMPMSMs). The authors attempted to minimize the cost and THD while maximizing the efficiency. Their results were verified via FEA. Moreover, they employed the same method for Interior PMM (IPMM) [34] and minimized torque ripple and THD. FEA was also used in this study for verification. Semon et al. [35] employed Response Surface Methodology (RSM) to optimize the rotor configuration of a V-type IPM Synchronous Motor (IPMSM). They attempted to minimize THD while keeping the airgap magnetic flux density in the desired range. Zhu et al. [36] studied the design optimization of a PMSG for a wind power generator using Taguchi method to improve the voltage regulation rate and THD. Karimpour et al. [37] applied FEA and Taguchi concurrently to optimize the design of an IPMSG to improve the efficiency, THD, and amplitude of induction EMF. In addition to these studies, a review study that was presented by Fallows et al. [38] summarizes the related literature well and it represents a remarkable study that deserves further examination.

Soft computing is an emerging technology that has widely been used to solve NP-hard problems (such as design problems). Most of these techniques are basically inspired by nature or collective behavioral patterns. The advent of soft computing to the computing world has been marked by research on machine learning and extended to Artificial Neural Networks (ANN), fuzzy logic, and GA. Then, soft computing has become inclusive enough to cover swarm intelligence like PSO. As technology gains further ground with time, it becomes quite important to find a novel solution to design optimization problems and upgrade the conventional methods. Today, new soft computing methods presented in the literature include Grey Wolf Optimizer (GWO), Bat Algorithm (BA), Whale Optimization Algorithm (WOA), Social Group Optimization (SGO), etc. All of the algorithms that are currently used by engineers and spread widely in 
Table 1. Materials for SG.

\begin{tabular}{lll}
\hline Components & Type of material & Description \\
\hline Windings & Standard copper & All winding material \\
Core (rotor \& stator) & Si-Fe lamination & M530-50A \\
Insulation class & $\mathrm{F}$ & Maximum $140^{\circ} \mathrm{C}$ \\
\hline
\end{tabular}

the industry were initially produced in the academic community. In addition, their performance in solving different types of problems was presented in academic studies and, then, turned into known methods. In this study, GWO was used for the optimization. GWO is a relatively new algorithm in this field. Publications that have demonstrated the performance of GWO in electric machine design problems contribute to the widespread use of such new-generation effective algorithms in this area, as was the case with GA and PSO before.

GWO is one of the recent and very effective swarm-based optimization algorithms. The objective of this study is to minimize the load of voltage THD on the SG by finding optimum levels of slot pitch, center slot pitch, and damper width with GWO. To this end, Maxwell simulation for several combinations of different levels of slot pitch, center slot pitch, and damper width is performed and the voltage THD is measured using Maxwell (in this study, all THD values indicate voltage harmonics). Then, the mathematical relation between the THD and the factors is determined via regression equations. Finally, GWO is employed to optimize this regression equation to determine the optimum factor levels so as to minimize the voltage THD. GWO has not been previously used in the literature for damper winding optimization and THD minimization. This is the primary novelty of this study.

Another novelty involves factor combination, which is used for optimization. In the related literature, slot pitch, center slot pitch, and damper width have not been used concurrently for minimizing THD, previously. The results of this study illustrate the relation between these factors and THD minimization. This is part of the contribution made by this study to the literature. This study differs from its counterparts in the literature in terms of (a) demonstrating the effect of slot pitch, center slot pitch, and damper width factors on design optimization with minimum production line adjustments for SGs produced under mass production conditions and (b) showing the effectiveness of using regression and GWO in optimization.

The motivational objective of this study is to demonstrate how to effectively minimize THD by optimizing such parameters as slot pitch, center slot pitch, and damper width via a few experimental runs. An attempt is made to prove that even the changes made to the mentioned factors have a positive effect on THD. GA and PSO are widely used for THD optimization.
Moreover, a limited number of studies have used ALO, AMBS, CSA, ANN, and fuzzy for THD optimization. However, GWO has not been previously applied to SG design to minimize THD. Demonstration of the effectiveness of GWO represents the second motivation of this research.

The importance of this research lies in the attempt to illustrate how the performance of SG can be improved by making minimal changes to the production line adjusted to mass production, without changing the outer diameter and dimensions of SG.

\section{Structure of the SG}

In this paper, a $60 \mathrm{kVA}$ AVR controlled SG is designed with a rated power factor of 0.8 . The rated voltage for this generator is $400 \mathrm{~V}$. This generator has a 4-pole rotor that gives $50 \mathrm{~Hz}$ at $1500 \mathrm{rpm}$. The SG materials are listed in Table 1.

According to Table 1, M530-50A lamination is used for both rotor and stator, and this core has Class$\mathrm{F}$ insulation between core and windings. The structure of the current SG to be optimized is presented in Figure 1.

The design parameters of the SG given in Figure 1 are shown in Table 2. During Maxwell simulations, the design parameters, except the slot pitch, center slot pitch, and damper width, given in Table 2 are kept constant.

In this paper, slot pitch, center slot pitch, and damper width are considered with the aim of minimizing the THD of SG. In the current rotor design of $\mathrm{SG}$, the used parameters include slot pitch $=8 \mathrm{deg}$., center slot pitch $=25$ deg., and damper width $=6$ $\mathrm{mm}$. In addition, the voltage THD is calculated as 2.95 and 2.8 based on Maxwell simulation result and the real product, respectively. The difference between
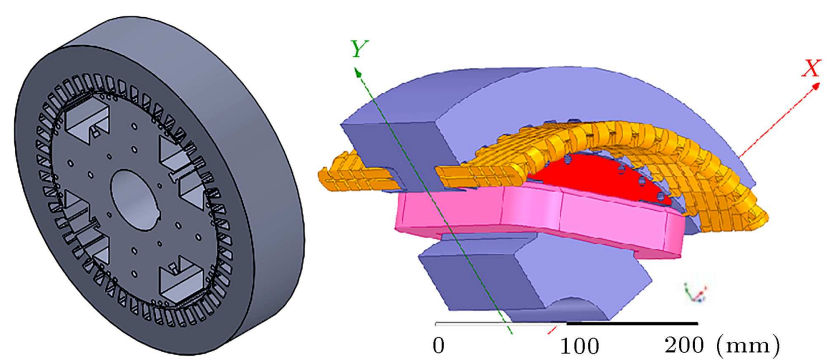

Figure 1. General structure of the current SG. 
Table 2. Design parameters of SG.

\begin{tabular}{ccccc}
\hline Name & Unit & Value & Description & Part \\
\hline Inner $\phi$ & $\mathrm{mm}$ & 299.5 & Core diameter (gap side) & Stator \\
Outer $\phi$ & $\mathrm{mm}$ & 440 & Core diameter (yoke side) & Stator \\
Length & $\mathrm{mm}$ & 130 & Length of core & Stator \\
Skew width & units & 1 & Range \# of slot & Stator \\
Slots & units & 60 & \# of slots & Stator \\
Slot type & N/A & 3 & Circular (slot type: 1 to 6) & Stator \\
Hs0 & $\mathrm{mm}$ & 1 & Slot opening height & Stator \\
Hs2 & $\mathrm{mm}$ & 1.35 & Slot height & Stator \\
Bs0 & $\mathrm{mm}$ & 3.6 & Slot opening width & Stator \\
Bs1 & $\mathrm{mm}$ & 13 & Slot width & Stator \\
Bs2 & $\mathrm{mm}$ & 13 & Slot width & Stator \\
Rs & $\mathrm{mm}$ & 2.5 & Slot bottom radius & Stator \\
Inner $\phi$ & $\mathrm{mm}$ & 110 & Core diameter (gap side) & Rotor \\
Outer $\phi$ & $\mathrm{mm}$ & 298 & Core diameter (yoke side) & Rotor \\
Length & $\mathrm{mm}$ & 130 & Core length & Rotor \\
Poles & - & 4 & \# of poles & Rotor \\
Pole-shoe width & $\mathrm{mm}$ & 91 & One pole max width & Rotor \\
Pole-shoe height & $\mathrm{mm}$ & 43 & One pole max width & Rotor \\
Pole-body width & $\mathrm{mm}$ & 150 & One pole max width & Rotor \\
Pole-body height & $\mathrm{mm}$ & 26 & One pole max width & Rotor \\
\# of dampers & $\mathrm{Units}$ & 6 & Damper winding \# per pole & Rotor \\
\hline
\end{tabular}

the expected and observed values results from the labor-intensive manufacturing operations of the firm. Very low harmonic value is ideal for terrestrial communication modules used in military projects, mobile radar towers, generators that supply missile ramps, and generators as power sources for unmanned aerial systems and marine power systems.

To provide data for regression modeling and GWO optimization, different levels of slot pitch, center slot pitch, and damper width are determined via experimental design and then, these experiments are run in Maxwell simulations. Finally, GWO algorithm is employed to optimize the structure of an SG rotor design.

\section{Regression modelling}

Upon finding the relationship between factors and THD based on the results of experiments (Maxwell simulation results) obtained for different combinations of factors in the offline mode and by searching for a mathematical function using GWO, the optimum levels of the factors are obtained. Then, this target value can be confirmed again in the Maxwell environment. In this paper, regression modeling is used to determine the mathematical relation between the THD and the factors having an effect on THD (slot pitch, center slot pitch, and damper width). Relevant literature pieces concluded the nonlinear relationship between the SG rotor design parameters and the THD. Moreover, this finding is confirmed by our previous trials. For this reason, a second-order regression model is used. A general representation of the model is given in $\mathrm{Eq}$. (1). This model will be calculated based on the Maxwell simulation results presented in Section 5 .

$$
Y=\beta_{0}+\sum_{i=1}^{k} \beta_{i} X_{i}+\sum_{i=1}^{k} \beta_{i i} X_{i}^{2}+\sum_{i<j}^{n} \beta_{i j} X_{i} X_{j}+\varepsilon,
$$

$Y$ is the value of THD, $X$ terms $\left(X_{i}\right.$ : linear terms, $X_{i}^{2}$ : quadratic terms, and $X_{i} X_{j}$ : interaction terms) are the values of the factors (slot pitch, center slot pitch, and damper width), $\beta$ terms $\left(\beta_{0}, \beta_{i}, \beta_{i i}\right.$, and $\left.\beta_{i j}\right)$ are the coefficients of the regression model, and $\varepsilon$ is residual [39]. It is possible to write the matrix notation for this model as:

$$
Y=\beta X+\varepsilon .
$$

According to this equation, $Y$ represents the matrix composed of the observed responses and $X$ represents the matrix composed of the factor values. $\beta$ matrix is composed of $\beta$ terms (regression model coefficient given in Eq. (1)) and calculated using Eq. (3). The 1st column of $X$ matrix is composed of 1 s for the constant 
term $\left(\beta_{0}\right)$ of the model and, then, it is arranged in order to have columns to contain all terms in the model. $\varepsilon$ is composed of the residual terms. $\beta$ matrix can be calculated by Eq. (3) [39]:

$$
\beta=\left(X^{T} X\right)^{-1}\left(X^{T} Y\right) \text {. }
$$

Upon examining the data given in Section 5 , one can realize that an $X$ matrix with dimensions of $12 \times 10$ will be obtained for 12 runs and 10 model coefficients $(\beta)$. After calculating the model parameters, coefficient of determination $\left(R^{2}\right)$ is computed. It is used to measure the level of the strength between the regression model and the factors.

$$
R^{2}=\frac{\beta^{T} X^{T} Y-n \bar{Y}^{2}}{Y^{T} Y-n \bar{Y}^{2}}
$$

In this equation, $n$ represents the number of experimental runs (number of observations) and $\bar{Y}$ is the mean value for the observed values. $R^{2}$ is desired to be closer to 1 (which means $100 \%$ ). It is implied that that the factors used in modeling are sufficient to explain the change in $Y$. The next step is determining if the model is significant or not. To this end, "analysis of variance (ANOVA)" is used. Minitab, which is a well-known statistical package, is used to conduct calculations. An approach namely " $p$-value" is used to perform decisionmaking. ANOVA is one of the most widely used statistical hypothesis tests to determine if the regression model is significant or not. By using ANOVA, two hypotheses are tested $\left(H_{0}\right.$ : null hypothesis and $H_{1}$ : alternative hypothesis). If $H_{0}$ is true (it means regression model is insignificant), then the " $p$-value" is greater than $\alpha$ (type-I error) (or the test statistic which is calculated from the observations $\left(F_{0}\right)$ is lower than the critical value from $F$-statistical table $\left(F_{\alpha, m-1, N-m}\right)$, where $m$ is the number of estimated regression coefficients and $N$ is the number of experimental runs). To be able to use the calculated model, $H_{0}$ must be rejected (meaning that $H_{1}$ is accepted). In this case, the $p$-value is less than $\alpha$ (or $F_{0}$ is greater than $F_{\alpha, m-1, N-m}$ ). A general representation of ANOVA table is presented in Table 3 [39]. In this table, df, SS, and MS denote "degrees of freedom", "Sum of Squares", and "Mean Squares", respectively.

This study enjoys a confidence level of $95 \%$ (in other words, $\alpha=0.05(5 \%))$. Following the completion of the regression modeling phase, GWO was used for minimizing the voltage THD by calculating the optimum factor levels for slot pitch, center slot pitch, and damper width.

\section{GWO algorithm}

GWO algorithm is a metaheuristic optimization technique that is inspired by nature and presented by Mirjalili et al. [40]. It simulates the swarm behavior of grey wolves. It is also known as a swarm intelligence method. GWO has been applied to several optimization problems in the literature. In this study, GWO algorithm is employed to optimize the factor levels of slot pitch, center slot pitch, and damper width to minimize the THD of an SG. If the regression model (evaluated in Section 3 ) is significantly viable according to the ANOVA results, then the GWO will be used for calculating the optimum factor levels using this regression equation.

The rationale behind the use of GWO algorithm is to mimic the superior characteristics, social hierarchy, and hunting behaviors of the grey wolves for solving the optimization problems. This algorithm proposes 4 types of wolves in a pack in nature [40]. Their hierarchical order involves alphas $(\alpha)$, betas $(\beta)$, and omegas $(\omega)$. Moreover, there are deltas $(\delta)$. Deltas are the subordinates and are not alpha, beta, or omega. Deltas must submit to alphas and betas, as omegas do. However, they have control over the omegas. Delta wolf group is composed of the wolfs which are categorized into 5 types: (i) the wolves responsible for watching (scouts), (ii) those protecting the wolf pack (sentinels), (iii) experienced wolves (the wolves that used to be alpha or beta previously; elders), (iv) the wolves providing food for the pack (hunters), and (v) those responsible for the wounded, ill, and weak wolves in the pack (caretakers) [40,41].

If we translate this nature-inspired terminology into math and optimization terminology, the solutions of the optimization problem will be defined as alpha, beta, delta, or omega. The positions of the wolves (optimum factor levels of slot pitch, center slot pitch, and damper width for this study) are updated by GWO algorithm during the discovery. This is performed by considering the current position of the wolves $\alpha, \beta$, and $\delta$. This is the standard progress of a metaheuristic optimization technique. To calculate the optimum response value (minimum THD), the main steps of the algorithm implementation given below are performed orderly: (i) encircling, (ii) hunting, (iii) attacking, and (iv) searching. In other words, prey is the minimum THD value for this study. Mirjalili et al. [40] and Ileri et al. [41] defined the first 3 fittest solutions as alpha, beta, and delta, respectively. In this case,

Table 3. ANOVA table.

\begin{tabular}{lcccc}
\hline \multicolumn{1}{c}{ Source } & $\boldsymbol{d} \boldsymbol{f}$ & $\boldsymbol{S} \boldsymbol{M}$ & $\boldsymbol{M S}=\boldsymbol{S} \boldsymbol{S} \boldsymbol{d} \boldsymbol{f}$ & $\boldsymbol{F}$ \\
\hline Regression & $m-1$ & SS treatments $\left(S S_{T r}\right)$ & $M S_{T r}$ & $F_{o}=\left(M S_{T r} / M S_{E}\right)$ \\
Residual error & $N-m$ & $S S$ error $\left(S S_{E}\right)$ & $M S_{E}$ & \\
\hline
\end{tabular}


the rest of the solutions are defined as the omega. GWO performs optimization (hunting) via alpha, beta, and delta wolves. These three fittest wolves, i.e., the three fittest solutions, are followed by omegas. In a metaheuristic optimization technique, the optimum result is searched for in a solution space (usually called population). This wolf pack generally makes up this population. The first step for GWO is hunting. In this step, the wolves encircle prey during the hunt. The mathematical representation of this behavior is simulated by Eqs. (5) and (6) [40,41]:

$$
\begin{aligned}
& \vec{D}=\left|\vec{C} \cdot \overrightarrow{X_{p}}(t)-\vec{X}(t)\right|, \\
& \vec{X}(t+1)=\overrightarrow{X_{p}}(t)-\vec{A} \cdot \vec{D} .
\end{aligned}
$$

Position vector of the prey is represented by $\overrightarrow{X_{p}}(t) . \vec{X}$ indicates the position vector of a grey wolf. $\vec{C}$ and $\vec{A}$ are coefficient vectors:

$$
\begin{aligned}
& \vec{A}=2 \vec{a} \cdot \overrightarrow{r_{1}}-\vec{a}, \\
& \vec{C}=2 \overrightarrow{r_{2}} .
\end{aligned}
$$

The components of $\vec{a}$ are linearly reduced from 2 to 0 during the iterations. $r_{1}$ and $r_{2}$ are random vectors in $[0,1]$. The alpha, beta, and delta wolves can give important information on the potential position (optimum factor levels of slot pitch, center slot pitch, and damper width) of a prey (minimum THD). Therefore, in order to realize them as guides for the rest of the solutions, the GWO algorithm must memorize the positions of alpha, beta, and delta (the first three best solutions obtained so far). This is the second step of this algorithm. In this regard, Eqs. (9)-(11) given below are proposed $[40,41]$ :

$$
\begin{aligned}
& \overrightarrow{D_{\alpha}}=\left|\overrightarrow{C_{1}} \cdot \overrightarrow{X_{\alpha}}-\vec{X}\right|, \quad \overrightarrow{D_{\beta}}=\left|\overrightarrow{C_{2}} \cdot \overrightarrow{X_{\beta}}-\vec{X}\right| \\
& \overrightarrow{D_{\delta}}=\left|\overrightarrow{C_{3}} \cdot \overrightarrow{X_{\delta}}-\vec{X}\right|, \\
& \overrightarrow{X_{1}}=\overrightarrow{X_{\alpha}}-\overrightarrow{A_{1}} \cdot\left(\overrightarrow{D_{\alpha}}\right), \overrightarrow{X_{2}}=\overrightarrow{X_{\beta}}-\overrightarrow{A_{2}} \cdot\left(\overrightarrow{D_{\beta}}\right) \\
& \overrightarrow{X_{3}}=\overrightarrow{X_{\delta}}-\overrightarrow{A_{3}} \cdot\left(\overrightarrow{D_{\delta}}\right), \\
& \vec{X}(t+1)=\frac{\overrightarrow{X_{1}}+\overrightarrow{X_{2}}+\overrightarrow{X_{3}}}{3} .
\end{aligned}
$$

The grey wolves finish the hunt by attacking the prey when the movement is halted (exploitation). In the third step of GWO implementation, the value of $\vec{a}$ decreases. This mathematically simulates the behavior of the wolves approaching the prey. $\vec{A}$ fluctuates in the range of $[-a, a]$ and it is reduced by $\vec{a}$. The pack attack the prey if the random values of $\vec{A}$ are in the range of $[-1,1][40,41]$.

In addition to these three main steps, the wolf pack search the area with the help of alpha, beta, and delta positions. When looking for prey, they divide and conquer. This is necessary to avoid trapping in local optimum and to seek a global optimum solution. For this purpose, $\vec{A}$ is utilized randomly and its value is less than -1 and greater than 1 . The search agent diverges from the prey in this way and GWO searches the area globally (exploration). Another component of the algorithm that favors exploration is $\vec{C}$. It involves random values from the $[0,2]$ interval and provides random weights for prey. In this way, it is possible to emphasize $(C>1)$ or de-emphasize $(C<1)$ the effect of prey in defining the distance stochastically. In addition, the local optimal solution is obstructed by facilitating the exploration [40, 41]. As a suggestion, the study of Mirjalili et al. [40] should be read in detail for more information on the operation of the GWO algorithm.

\section{Numerical results and discussions}

There are many design parameters that affect the THD performance of SG and this issue is investigated in this study (see Table 2). The real attempt of this research is to optimize some of the rotor design parameters of SG (slot pitch, center slot pitch, and damper width) to get the minimum voltage THD and acceptable magnetic flux density. Regression modeling is employed to determine the mathematical relationship between the rotor design parameters (factors) and THD (response). Then, GWO is applied to this regression equation to minimize the THD. The optimization process flowchart is presented in Figure 2. The levels of factors are presented in Table 4. Figure 3 gives the structure of the current SG with its dimensions.

In the case of GWO optimization, it is preferable to utilize the regression equation calculated with coded factor levels [40]. Therefore, uncoded and coded factor levels are presented in Table 5. The THD values presented in Table 5 are calculated under nominal load conditions. The coding is performed via Eq. (12):

$$
X_{\text {coded }}=\frac{X_{\text {uncoded }}-\left(\left(X_{\max }+X_{\min }\right) / 2\right)}{\left(X_{\max }-X_{\min }\right) / 2} .
$$

Table 4. Factor levels.

\begin{tabular}{lccccccc}
\hline & & & \multicolumn{4}{c}{ Levels } \\
\cline { 4 - 7 } Factors & Symbol & Unit & $\mathbf{1}$ & $\mathbf{2}$ & $\mathbf{3}$ & $\mathbf{4}$ \\
\hline Slot Pitch (SP) & $X_{1}$ & Degree & 5 & 7 & 9 & - \\
Center Slot Pitch (CSP) & $X_{2}$ & Degree & 20 & 22 & 25 & 30 \\
Damper Width (DW) & $X_{3}$ & mm & 6 & 7 & 8 & - \\
\hline
\end{tabular}




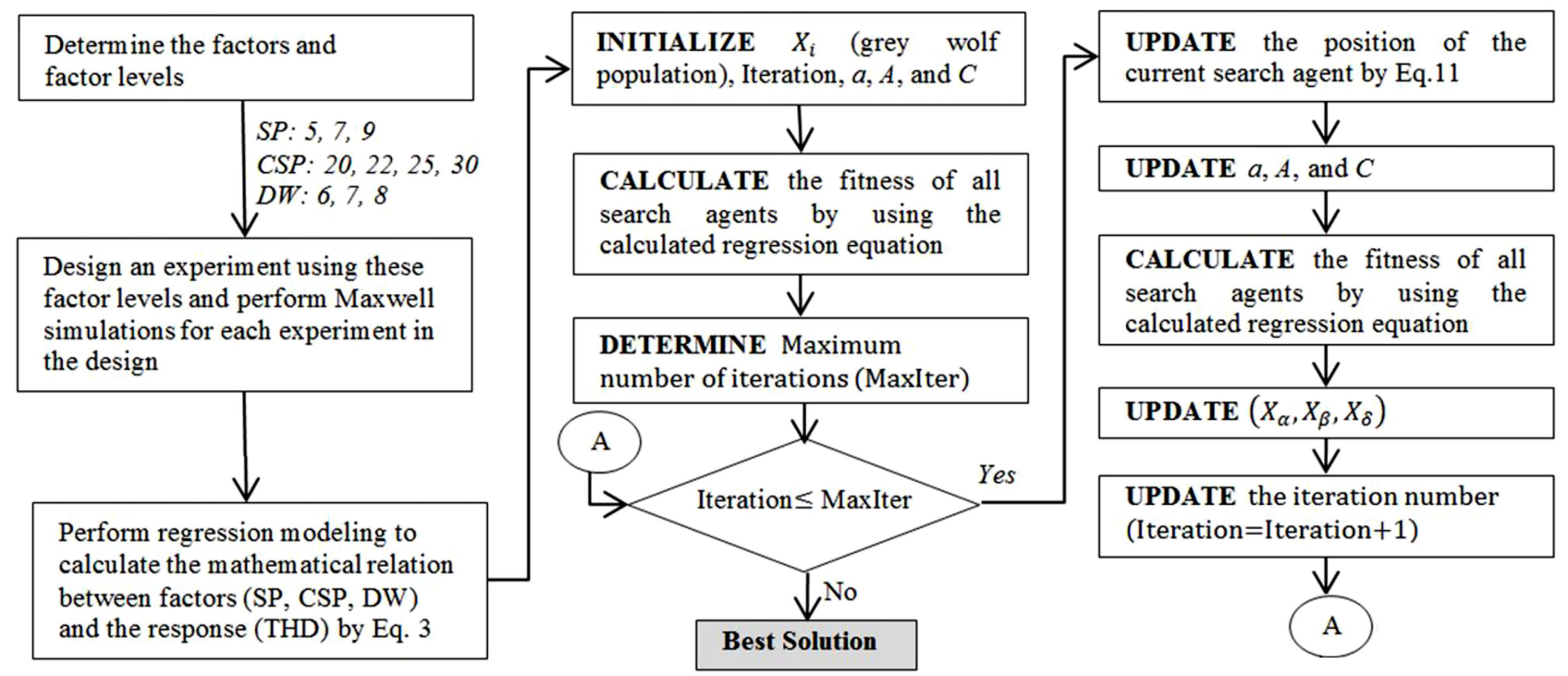

Figure 2. Flowchart of the optimization process.

The regression model is derived by second-order regression according to several preliminary trials and literature review. For this purpose, 12 experimental runs composed of different combinations of factor
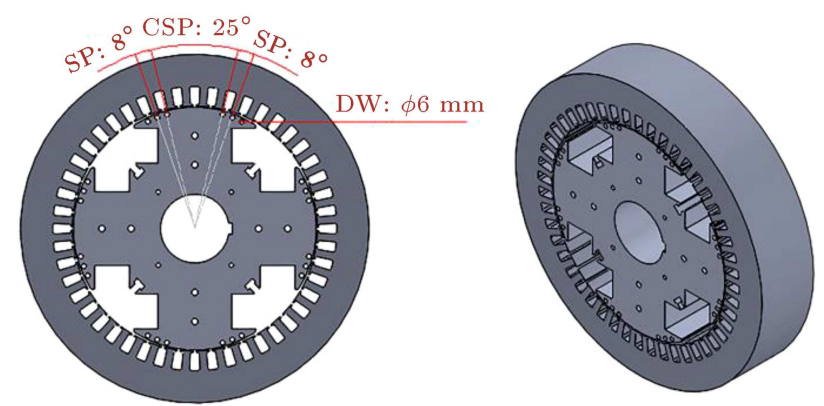

Figure 3. Structure of the existing SG (Maxwell rotor design dimensions).

Table 5. Maxwell simulation results for the experimental design.

\begin{tabular}{cccccccc}
\hline & \multicolumn{3}{c}{$\begin{array}{c}\text { Factors } \\
\text { (uncoded levels) }\end{array}$} & \multicolumn{2}{c}{$\begin{array}{c}\text { Factors } \\
\text { (coded levels) }\end{array}$} & $\begin{array}{c}\text { Response } \\
\text { (THD) }\end{array}$ \\
\cline { 2 - 8 } Run & $\boldsymbol{X}_{\mathbf{1}}$ & $\boldsymbol{X}_{\mathbf{2}}$ & $\boldsymbol{X}_{\mathbf{3}}$ & $\boldsymbol{X}_{\mathbf{1}}$ & $\boldsymbol{X}_{\mathbf{2}}$ & $\boldsymbol{X}_{\mathbf{3}}$ & $\boldsymbol{Y}_{\boldsymbol{i}}$ \\
\hline 1 & 5 & 20 & 6 & -1 & -1 & -1 & 4.96 \\
2 & 9 & 20 & 6 & 1 & -1 & -1 & 2.57 \\
3 & 5 & 30 & 6 & -1 & 1 & -1 & 4.60 \\
4 & 9 & 22 & 6 & 1 & -0.6 & -1 & 3.32 \\
5 & 5 & 20 & 8 & -1 & -1 & 1 & 4.44 \\
6 & 9 & 20 & 8 & 1 & -1 & 1 & 9.12 \\
7 & 9 & 25 & 7 & 1 & 0 & 0 & 7.72 \\
8 & 7 & 20 & 7 & 0 & -1 & 0 & 2.66 \\
9 & 7 & 30 & 7 & 0 & 1 & 0 & 2.81 \\
10 & 7 & 25 & 6 & 0 & 0 & -1 & 2.07 \\
11 & 7 & 25 & 8 & 0 & 0 & 1 & 3.06 \\
12 & 7 & 25 & 7 & 0 & 0 & 0 & 3.21 \\
\hline
\end{tabular}

levels are provided by Maxwell simulations. Minitab (a statistical package program) is employed to calculate the regression coefficients. The original model is given in Eq. (13):

$$
\begin{aligned}
& \text { THD } D_{\text {uncoded }}=26.3392951969-18.6544900489102 X_{1} \\
& +1.1692507776 X_{2}+6.4386851996 X_{3} \\
& +0.7412555503 X_{1}^{2}-0.0122433565 X_{2}^{2} \\
& \quad-0.5260895485 X_{3}^{2}+0.1127366567 X_{1} X_{2} \\
& +0.8989042813 X_{1} X_{3}-0.1921789844 X_{2} X_{3} \cdot(13)
\end{aligned}
$$

In order to use this equation in GWO, the factors must be coded in value between -1 and 1 . The equation employed for GWO using the coded values in Table 5 is given as follows:

$$
\begin{aligned}
& \text { TH } D_{\text {coded }}=3.0948693849+1.6676680866 X_{1} \\
& +0.0049332837 X_{2}+0.5612868799 X_{3} \\
& +2.9650222012 X_{1}^{2}-0.3060839137 X_{2}^{2} \\
& \quad-0.5260895485 X_{3}^{2}+1.1273665675 X_{1} X_{2} \\
& +1.7978085627 X_{1} X_{3}-0.9608949219 X_{2} X_{3} \cdot(14)
\end{aligned}
$$

The $R^{2}$ statistics associated with the given model is 99.84. The model significance is tested using ANOVA. The result of ANOVA is given in Table 6 (confidence level of 95\%). The surface plots for the THD with uncoded factor levels are given in Figures 4-6.

According to the presented results, it can be concluded that the regression model is significant. 
Table 6. ANOVA table.

\begin{tabular}{lccccc}
\hline Source of variation & df & SS & MS & $\boldsymbol{F}_{\mathbf{0}}$ vs $\boldsymbol{F}_{\mathbf{0 . 0 5 , 9 , 2}}$ & $\begin{array}{c}\boldsymbol{p} \text {-value vs } \\
\boldsymbol{\alpha}=\mathbf{0 . 0 5}\end{array}$ \\
\hline Regression & 9 & 51.86 & 5.762 & $140.53>19.385$ & $0.007<0.05$ \\
Residual error & 2 & 0.082 & 0.041 & & \\
\hline
\end{tabular}

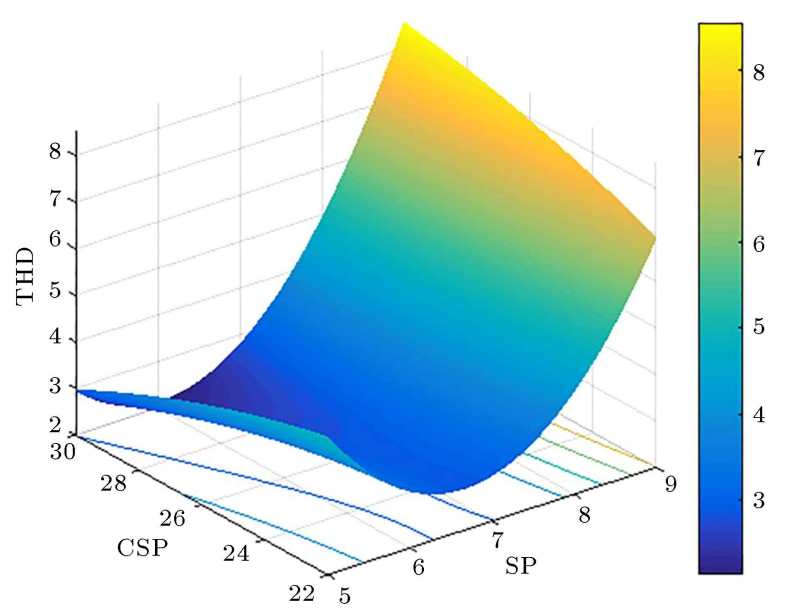

Figure 4. Surface plot for THD versus CSP and SP.

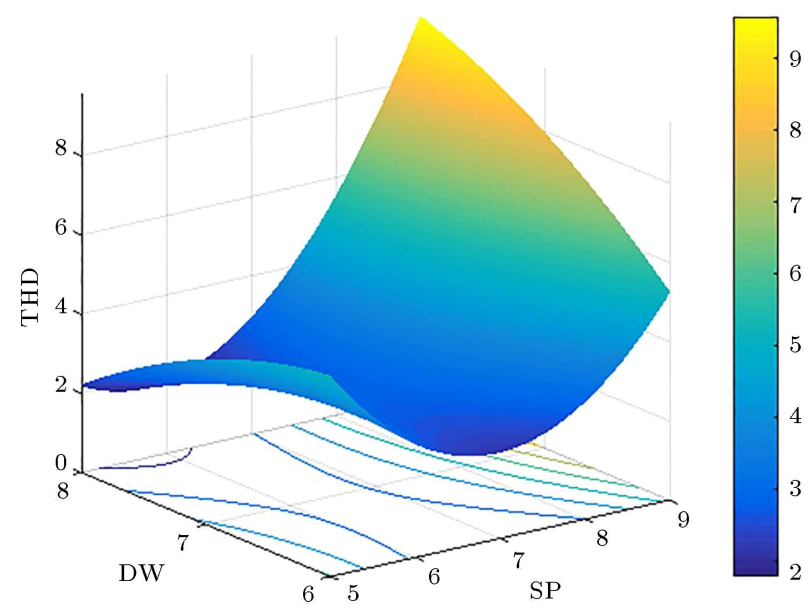

Figure 5. Surface plot for THD versus DW and SP.

The prediction performance of the regression model is given in Table 7 . Maxwell simulations are the observed responses $\left(Y_{i}\right)$, while the expected responses $\left(\hat{Y}_{i}\right)$ are predicted by regression equation. Prediction Error $(\mathrm{PE})$ is the error between the Maxwell simulations and the regression model predictions. PE (\%) for the $i$ th observation is calculated by the formula given in Eq. (15):

$$
P E(\%)=\frac{\left|Y_{i}-\hat{Y}_{i}\right|}{\hat{Y}_{i}} 100 .
$$

According to Table 7, the regression model is in good fit with the given observations (with a PE $(\%)<6 \%$ ). $R^{2}$ value, ANOVA results, and comparisons given in Table 7 prove that this mathematical model can be

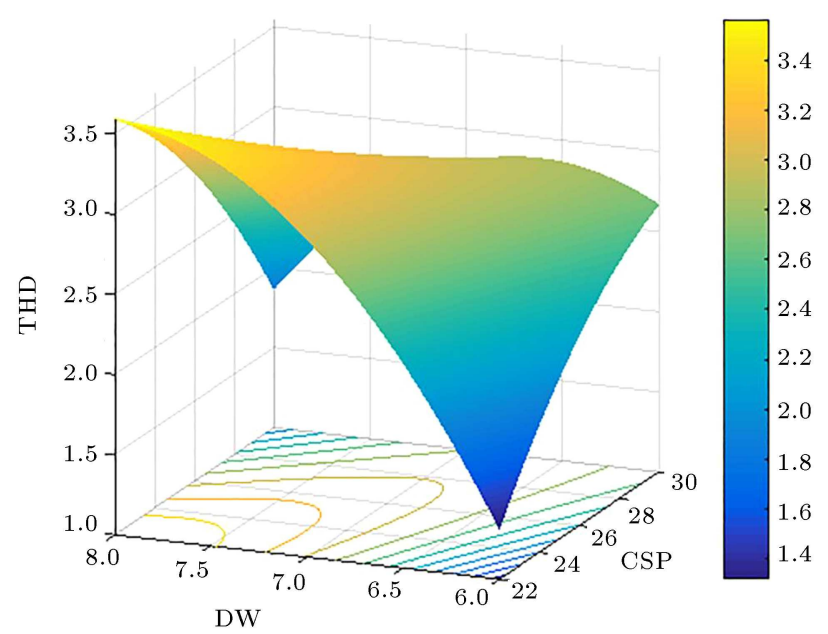

Figure 6. Surface plot for THD versus CSP and DW.

considered for the optimization. MATLAB program is used for coding GWO algorithm. The GWO is run on a PC (Intel Core-i5, RAM: 4 GB). The use of the algorithm helped the decision to utilize 45 search agents. The maximum number of 10000 iterations is set. The GWO parameters (such as number of search agents, etc.) and the number of iterations was determined through a set of preliminary trials. The problem was modeled as a constrained continuous optimization problem (using the previously identified regression Eq. (14)) as given below and, then, the

Table 7. Performance of the mathematical model.

\begin{tabular}{cccc}
\hline Run & $\boldsymbol{Y}_{\boldsymbol{i}}$ & $\hat{\boldsymbol{Y}}_{\boldsymbol{i}}$ & $\boldsymbol{P} \boldsymbol{E}_{\boldsymbol{i}}(\boldsymbol{\%})$ \\
\hline 1 & 4.96 & 4.96 & 0.04 \\
2 & 2.57 & 2.44 & 5.19 \\
3 & 4.6 & 4.64 & 0.76 \\
4 & 3.32 & 3.48 & 4.50 \\
5 & 4.44 & 4.41 & 0.75 \\
6 & 9.12 & 9.08 & 0.41 \\
7 & 7.72 & 7.73 & 0.10 \\
8 & 2.66 & 2.78 & 4.45 \\
9 & 2.81 & 2.79 & 0.58 \\
10 & 2.07 & 2.01 & 3.11 \\
11 & 3.06 & 3.13 & 2.24 \\
12 & 3.21 & 3.09 & 3.72 \\
\hline
\end{tabular}


GWO algorithm was ran through this model.

$\operatorname{Min} T H D$ s.t. $X_{1} \in[-1,1] ; X_{2} \in[-1,1]$;

$$
X_{3} \in[-1,1] .
$$

The CPU time is calculated to be below 10 seconds. GWO algorithm evaluates the optimized factor levels as $X_{1}=5.451$ (coded value: -0.7745$), X_{2}=30$ (coded value: +1 ), and $X_{3}=8$ (coded value: +1 ). For this optimized factor level combination, the Maxwell simulation is performed and THD is calculated as 0.3951. This value is 6 times smaller than even the smallest value in the simulated data presented in Table 5. The structures of the optimized SG, voltage graph (for initial and optimized SGs), and magnetic flux distribution of the optimized SG are given in Figures 7-10, respectively.

The GWO results are also confirmed with GA, which is a widely used nature-inspired algorithm in the Electrical Engineering Society. Matlab is used for coding GA. GA is run for 100000 iterations. We determined GA parameters through some preliminary trials. The population size is selected as 80 . Moreover, the mutation and crossover rates are selected as 0.4 and 0.5 , respectively. GA is employed to calculate the
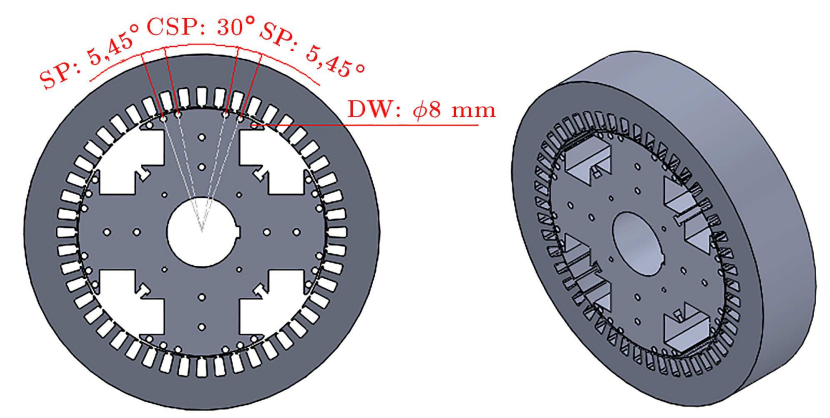

Figure 7. Structure of the optimized SG.

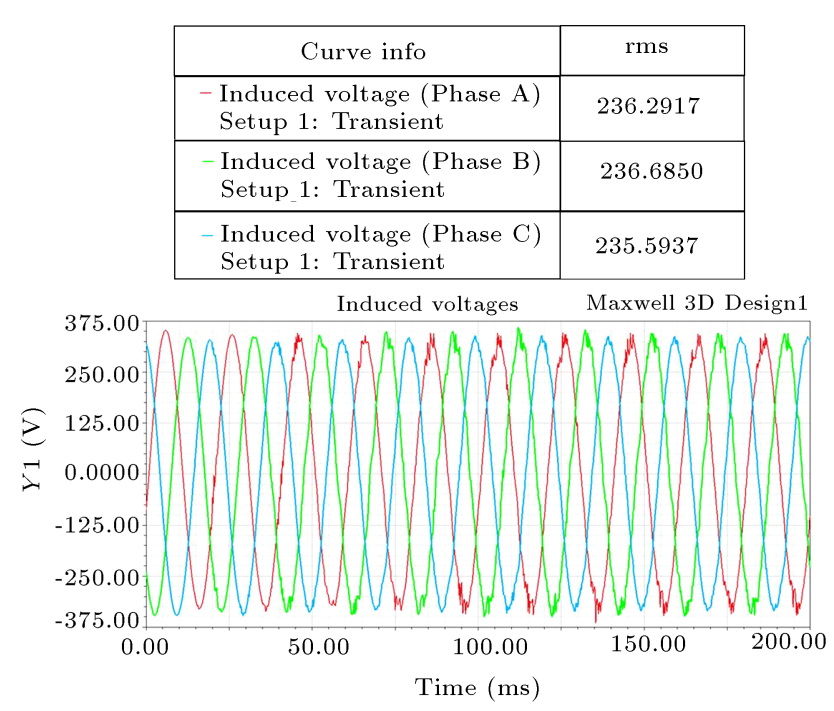

Figure 8. Voltage graph of the initial SG. optimized factor levels as $X_{1}=5.451$ (coded value: - 0.7745), $X_{2}=29.9995$ (coded value: +0.9999 ), and $X_{3}=7.9999$ (coded value: +0.9999 ). However, according to the serial production constraints, these coded values for $X_{2}$ and $X_{3}$ are rounded to +1 . As a result, the GWO and GA are found to be having almost the same optimum parameter levels. The advantage of GWO over GA in dealing with this problem is that it achieves appropriate results with far less iterations and without the need to round over optimum factor levels.

According to Figures 8 and 9, the THD is clearly optimized. In addition, it can be observed from Figure 10 that the magnetic flux density is in the acceptable limits. The main reason to provide a damper cage in the salient-pole SG is to prevent the distortion of voltage waveform. One of the major reasons for these

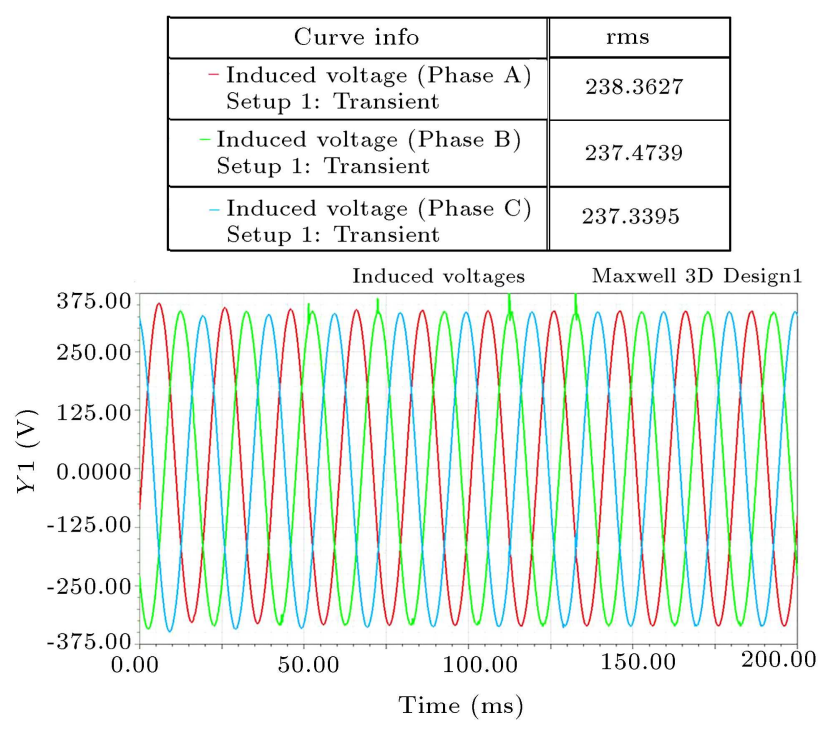

Figure 9. Voltage graph of the optimized SG.

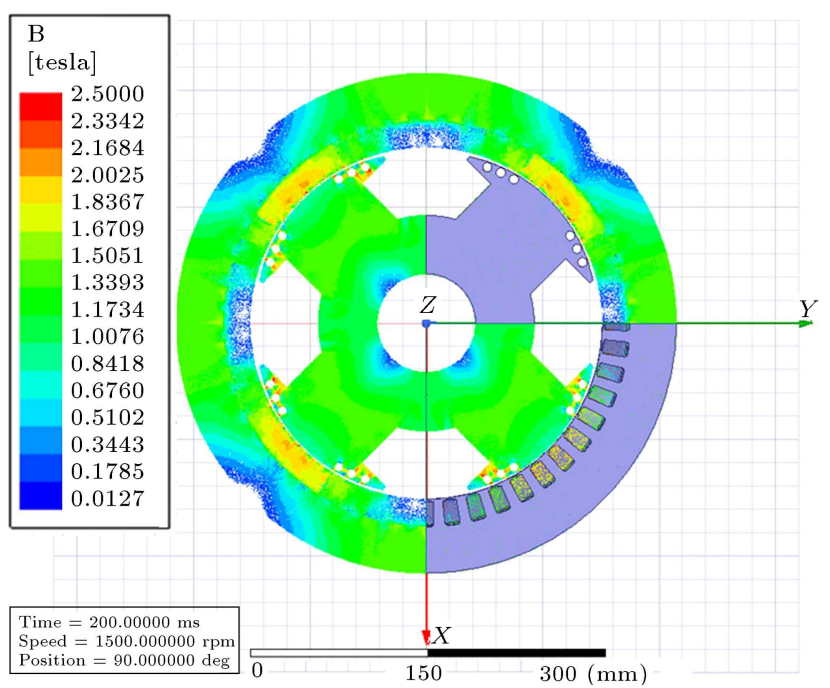

Figure 10. Magnetic flux distribution of the optimized SG. 
Table 8. Performance test results of the initial and optimized prototypes.

\begin{tabular}{|c|c|c|c|c|c|c|c|c|c|c|c|}
\hline \multirow[t]{2}{*}{ Load } & \multicolumn{3}{|c|}{ Voltage (Phase) } & \multicolumn{3}{|c|}{ Currents (Phase) } & \multicolumn{2}{|c|}{ Power } & \multirow[b]{2}{*}{$\operatorname{Cos} \varphi$} & \multirow[b]{2}{*}{$\begin{array}{c}\text { Rpm } \\
(\mathrm{r} / \mathrm{min})\end{array}$} & \multirow[b]{2}{*}{ THD } \\
\hline & U-V & U-W & V-W & I 1 & I 2 & I 3 & KW & KVA & & & \\
\hline \multicolumn{12}{|l|}{ Initial design } \\
\hline Load Step 1 & 400.6 & 400.5 & 400.9 & 28.40 & 28.10 & 28.40 & 14.42 & 16.77 & 0.86 & 1500 & 3.2 \\
\hline Load Step 2 & 400.3 & 400.1 & 400.7 & 61.60 & 59.90 & 61.20 & 33.85 & 41.80 & 0.81 & 1500 & 2.9 \\
\hline Load Step 3 & 399.5 & 400.0 & 401.6 & 83.80 & 80.90 & 83.40 & 45.20 & 56.50 & 0.80 & 1500 & 2.7 \\
\hline Load Step 4 & 399.6 & 400.0 & 401.8 & 92.90 & 89.00 & 92.30 & 48.90 & 62.70 & 0.78 & 1500 & 2.8 \\
\hline Load Step 5 & 399.5 & 399.9 & 401.8 & 105.80 & 102.50 & 105.2 & 56.55 & 71.60 & 0.79 & 1500 & 2.8 \\
\hline \multicolumn{12}{|c|}{ Optimized design } \\
\hline Load Step 1 & 400.3 & 400.2 & 400.6 & 28.90 & 28.40 & 28.70 & 17.02 & 19.80 & 0.86 & 1500 & 1.2 \\
\hline Load Step 2 & 399.8 & 399.9 & 400.2 & 52.40 & 51.60 & 51.70 & 29.70 & 35.90 & 0.83 & 1500 & 0.9 \\
\hline Load Step 3 & 398.1 & 399.1 & 400.9 & 84.40 & 83.50 & 80.70 & 45.40 & 57.20 & 0.79 & 1500 & 0.5 \\
\hline Load Step 4 & 397.5 & 398.7 & 400.8 & 93.90 & 93.10 & 89.70 & 49.40 & 63.70 & 0.78 & 1500 & 0.7 \\
\hline Load Step 5 & 397.2 & 398.5 & 400.4 & 103.50 & 102.20 & 98.80 & 57.50 & 70.20 & 0.82 & 1500 & 0.6 \\
\hline
\end{tabular}

harmonics is open slot structure typical of high-voltage machines. Therefore, by changing size, the place and number of damper windings can reduce the THD level of the alternator [23]. The results indicate that increase in DW absorbs the fugitive magnetic fluxes in the stator windings, reducing the formation of inverse EMF and harmonics. When DW increases and mounts close to each other (SP decreases), the probability of catching leakage fluxes increases. However, in case SP is less than 5.45 (damper windings approaching each other excessively), the positive effect on THD is reduced. Due to the internal resistance of copper damper rods, the effect on harmonics up to a certain diameter will be positive. Increased internal resistance of coppers will reduce the effect of damper rods on leakage flux. Therefore, it is a good limit for an $8 \mathrm{~mm}$ diameter for DW. CSP $=30$ is the upper limit value for this rotor design. If this value increases, damper windings cannot fit in the specified area.
IEEE STD 519-1992 standard requires $5 \%$ of the general system. This value is $8 \%$ for Pacific Corp standard [6]. The confirmation results indicate that the THD is 0.3951 for the optimized SG and this value is in accordance with the related standards.

The data in Table 8 show THD values under different loads with the application of the "gradual load test". During this test, alternators were connected to the test system and loaded with increasing values and the results were recorded. It is clear that the performance of the optimized alternator is better than the initial alternator performance. Real field test results and simulation results are relatively close to each other and at an acceptable level. As mentioned before, the difference between the expected and observed values is derived from the labor-intensive manufacturing operations of the firm. Moreover, Figure 11 shows a visual representation of the prototype produced at the end of optimization.

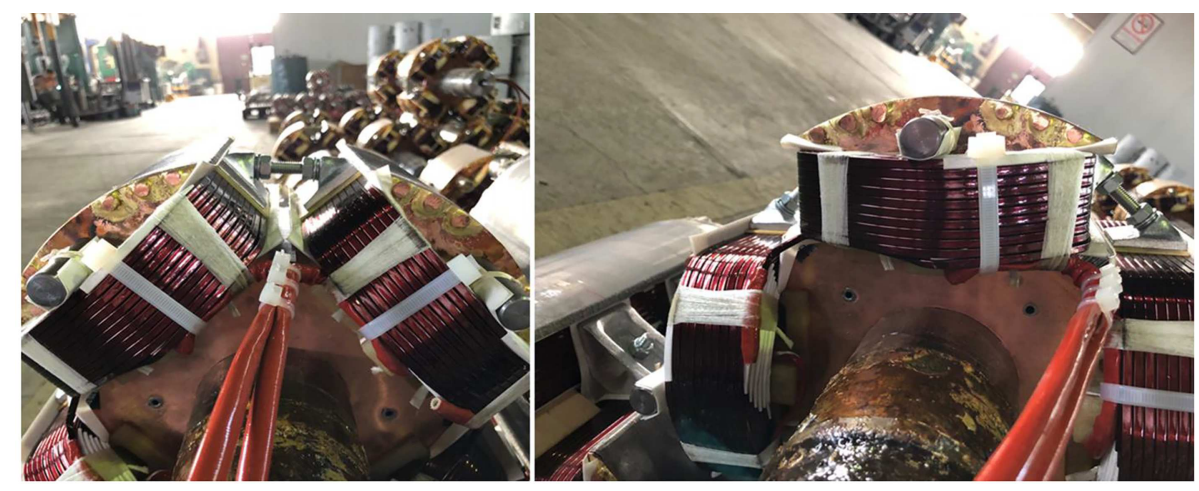

Figure 11. Visual of the prototype produced at the end of optimization. 


\section{Conclusion}

In this study, some of the design parameters for a $60 \mathrm{kVA} 1500 \mathrm{rpm}$ 4-pole brushless AVRcontrolledSynchronous Generator (SG) were optimized in order to minimize Total Harmonic Distortion (THD). GWO was used to calculate the optimum factor levels of the design parameters, namely SP, CSP, and DW. At first, levels for factors were determined. Then, regression modeling was employed for determining the mathematical relationship. Finally, Grey Wolf Optimizer (GWO) was utilized for optimization. The experimental results were measured with respect to the simulations conducted using Maxwell relations. The main drawback of producing so many SG prototypes - being uncertain - was eliminated. As a result, the THD of the SG was minimized to $0.3951 \%$ (by Maxwell simulations), which was very close to $0 \%$. Moreover, the distribution of the magnetic flux was kept in acceptable limits. In addition, a prototype of the optimized alternator design was produced and the THD value was found $0.8 \%$. Results indicated that this approach could be used for SG optimization. In addition, the results point to how well regression modeling adapts to the GWO optimization, depending on the numerical simulation. In this analysis, the THD could be minimized when too many SG design parameters were kept constant and only when some of the rotor design parameters were addressed. This study also investigated the damper windings of the alternator so as to avoid the design changes, which resulted in the new design of the product. The results of the optimized SG outperformed the current design of the company. The intended aim was to ensure that the serial production line layout and its operation would be minimally affected. In doing so, the constraint namely the redesigning of the assembly parts might affect the standard production, body design, cooling design, suitability for customer projects, and production difficulties and it was eliminated. The optimization results indicate that the GWO algorithm could be effectively used for design optimization of the SG in order to minimize THD. In future research, more other SG design parameters affecting THD might be considered for optimization. This research should be extended using additional design parameters affecting the THD of induced voltage determined by sensitivity analysis and the results will be confirmed by Finite Element Analysis (FEA).

\section{Acknowledgments}

We would like to thank Isbir Electric Company Research \& Development Department for giving us the opportunity to use its facilities and software. We also gratefully thank Mehmet Baki Dogru for his support.

\section{References}

1. Saha, S., Das, S., and Nandi, C. "Harmonics analysis of power electronics loads", International Journal of Computer Applications, 92, pp. 32-36 (2014).

2. Zaneta, E., Anton, B., and Mucha, M. "Harmonic distortion produced by synchronous generator in thermal power plant", 6th WSEAS International Conference on Power Systems, Lisbon, Portugal, Sep. pp. 22-24 (2006).

3. Arrillaga, J. and Watson, N.R., Power System Harmonics, 2nd Ed., John Wiley \& Sons, New Jersey, Hoboken, USA (2003).

4. Sayyah, A., Aflaki, M., and Rezazade, A.R. "Optimization of THD and suppressing certain order harmonies in PWM inverters using genetic algorithms", IEEE International Symposium on Intelligent Control, Munich, Germany, Oct. 4-6 (2006).

5. De Almeida, A.M.F., Pamplona, F.M.P., Braz, H.D.M., et al. "Multiobjective optimization for volt/THD problem in distribution system", 6th World Congress on Nature and Biologically Inspired Computing (NaBIC), Porto, Portugal, Jul 30-Aug. 01 (2014).

6. Pradigta, S.R.L., Asrarul, Q.O., Arief, Z., et al. "Reduction of total harmonic distortion (THD) on multilevel inverter with modified PWM using genetic algorithm", Emitter-International Journal of Engineering Technology, 5(1), pp. 91-118 (2017).

7. Rodriguez, J.L.D., Fernandez, L.D.P., and Penaranda, E.A.C. "Multiobjective genetic algorithm to minimize the THD in cascaded multilevel converters with $\mathrm{V} / \mathrm{F}$ control", 4th Workshop on Engineering Applications (WEA), Univ Tecnologica Bolivar, Cartagena, Colombia, Sep. 27-29 (2017).

8. Fernandez, L.D.P., Rodriguez, J.L.D., and Penaranda, E.A.C. "Optimization of the THD and the RMS voltage of a cascaded multilevel power converter", IEEE International Conference on Automation (ICA) / $23 r d$ Congress of the Chilean-Association-of-AutomaticControl (ACCA), Concepcion, Chile, Oct. 17-19 (2018).

9. Fernandez, L.D.P., Rodriguez, J.L.D., and Penaranda, E.A.C. "A multiobjective genetic algorithm for the optimization of the THD and the RMS output voltage in a multilevel converter with 17 levels of line voltage", IEEE Colombian Conference on Applications in Computational Intelligence (ColCACI), Barranquilla, Colombia, Jun. 5-7 (2019).

10. Booln, M.B. and Cheraghi, M. "THD minimization in a five-phase five-level VSI using a novel SVPWM technique", 10th International Power Electronics, Drive Systems and Technologies Conference (PEDSTC), Shiraz Univ, Shiraz, Iran, Feb. 12-14 (2019).

11. Alinejad-Beromi, Y., Sedighizadeh, M., and Sadighi, M. "A particle swarm optimization for sitting and sizing of distributed generation in distribution network to improve voltage profile and reduce THD 
and losses", 43rd International-Universities-PowerEngineering Conference, Padova, Italy, Sep. 1-4 (2008).

12. Gallardo, J.A.A., Rodriguez, J.L.D., and Garcia, A.P. "THD optimization of a single phase cascaded multilevel converter using PSO technique", Workshop on Power Electronics and Power Quality Applications (PEPQA), Bogota, Colombia, Jul. 6-7 (2013).

13. Kanth, D.S.K. and Lalitha, M.P. "Mitigation of real power loss, THD \& enhancement of voltage profile with optimal DG allocation using PSO \& sensitivity analysis", Annual International Conference on Emerging Research Areas - Magnetics, Machines and Drives (AICERA/iCMMD), Kottayam, India, Jul. 2426 (2014).

14. Memon, M.A., Memon, S., and Khan, S. "THD minimization from H-bridge cascaded multilevel inverter using particle swarm optimization technique", Mehran University Research Journal of Engineering and Technology, 36(1), pp. 33-38 (2017).

15. Dhanalakshmi, M.A., Ganesh, M.P., and Paul, K. "Analysis of optimum THD in asymmetrical H-bridge multilevel inverter using HPSO algorithm", 2nd International Conference on Intelligent Computing and Applications (ICICA), KCG Coll Technol, Chennai, India, Feb. 5-6 (2016).

16. Khanjanzadeh, A., Sedighizadeh, M., Rezazadeh, A., et al. "Using clonal selection algorithm for sitting and sizing of distributed generation in distribution network to improve voltage profile and reduce THD and losses", Int. Rev. Electr. Eng.-I, 6(3), pp. 1325-1331 (2011).

17. Francis, R. and Meganathan, D. "An improved ANFIS with aid of ALO technique for THD minimization of multilevel inverters", J. Circuit Syst. Comp., 27(12), article number: 1850193 (2018).

18. Khalid, S. and Verma, S. "THD and compensation time analysis of three-phase shunt active power filter using adaptive mosquito blood search algorithm (AMBS)", International Journal of Energy Optimization and Engineering (IJEOE), 8(1), pp. 25-46 (2019).

19. Knight, A.M., Karmaker, H., and Weeber, K. "Use of a permeance model to predict force harmonic components and damper winding effects in salientpole synchronous machines", IEEE T. Energy Conver., 17(4), pp. 478-484 (2002).

20. Rahimian, M.M. and Butler-Purry, K. "Modeling of synchronous machines with damper windings for condition monitoring", Proc. 2009 IEEE International Electric Machines and Drives Conference, pp. 577-584, Miami, FL (2009).

21. Traxler-Samek, G., Lugand, T., and Schwery, A. "Additional losses in the damper winding of large hydrogenerators at open-circuit and load conditions", IEEE T. Ind. Electron., 57(1), pp. 154-160 (2010).
22. Wallin, M., Bladh, J., and Lundin, U. "Damper winding influence on unbalanced magnetic pull in salient pole generators with rotor eccentricity", IEEE T. Magn., 49(9), pp. 5158-5165 (2013).

23. Nuzzo, S., Degano, M., Galea, M., et al. "Improved damper cage design for salient-pole synchronous generators", IEEE T. Ind. Electron., 64(3), pp. 1958-1970 (2017).

24. Elez, A., PetriniC, M., PetriniC, M., et al. "Salient pole synchronous generator optimization by combined application of slot skew and damper winding pitch methods", Progress in Electromagnetics Research $M$, 73, pp. 81-90 (2018).

25. Mandrile, F., Carpaneto, E., and Bojoi, R. "Virtual synchronous generator with simplified single-axis damper winding", 28th IEEE International Symposium on Industrial Electronics (IEEE-ISIE), Vancouver, Canada, Jun. 12-14 (2019).

26. Nuzzo, S., Bolognesi, P., Gerada, C., et al. "Simplified damper cage circuital model and fast analyticalnumerical approach for the analysis of synchronous generators", IEEE T. Ind. Electron., 66(1), pp. 83618371 (2019).

27. Jiji, K., Jayadas, N., and Babu, C. "FEM-based virtual prototyping and design of third harmonic excitation system for low-voltage salient-pole synchronous generators", IEEE T. Ind. Appl., 50(3), pp. 1829-1834 (2014).

28. Nuzzo, S., Galea, M., Gerada, C., et al. "Damper cage loss reduction and no-load voltage THD improvements in salient-pole synchronous generators", 8th IET International Conference on Power Electronics, Machines and Drives (PEMD 2016), Glasgow, UK, Apr. 19-21 (2016).

29. De la Cruz, J., Arredondo, J.M.R., and Delgado, J.A. "Strategies for improving voltage waveform of an outer rotor PMSG: no-load prototype testing", IEEE Lat. Am. T., 14(11), pp. 4547-4553 (2016).

30. Spargo, A., Ilie, S., and Chan, J. "Salient-pole rotor optimisations for synchronous generators using FEA software", 2017 IEEE Workshop on Electrical Machines Design, Control and Diagnosis (WEMDCD), Nottingham, UK, Apr. 20-21 (2017).

31. Wang, Y.L., Vakil, G., Nuzzo, S., et al. "Sensitivity analysis for performance and power density improvements in salient-pole synchronous generators", 2017 IEEE Workshop on Electrical Machines Design, Control and Diagnosis (WEMDCD), Nottingham, UK, Apr. 20-21 (2017).

32. Choi, G.S., Surname, J.H.J., and Hahn, S.C. "Characteristic analysis of synchronous generator according to the shape of slot", 21st International Conference on Electrical Machines and Systems (ICEMS), Jeju, South Korea, Oct 07-10 (2018).

33. Dai, L.T., Gao, J., Zhang, W.J., et al. "A genetic-Taguchi global design optimization strategy for surface-mounted PM machine", 22nd International Conference on Electrical Machines and Systems (ICEMS), Harbin, PRC, Aug. 11-14 (2019). 
34. Dai, L.T., Gao, J., Zhang, W.J., et al. "A geneticTaguchi global design optimization strategy for interior PM machine", 22nd International Conference on Electrical Machines and Systems (ICEMS), Harbin, PRC, Aug. 11-14 (2019).

35. Semon, A., Melcescu, L., Craiu, O., et al. "Design optimization of the rotor of a V-type interior permanent magnet synchronous motor using response surface methodology", 11th International Symposium on Advanced Topics in Electrical Engineering (ATEE), Bucharest, Romania, Mar. 28-30 (2019).

36. Zhu, J., Li, G.H., Cao, D., et al. "Voltage regulation rate and THD optimization analysis of coreless axial flux PM synchronous generator for wind power generation", IEEJ T. Electr. Electr., 14(10), pp. 1485-1493 (2019).

37. Karimpour, S.R., Besmi, M.R., and Mirimani, S.M. "Optimal design and verification of interior permanent magnet synchronous generator based on FEA and Taguchi method", Int. T. Electr. Energy, 30(11), Article Number: e12597 (2020).

38. Fallows, D., Nuzzo, S., Costabeber, A., et al. "Harmonic reduction methods for electrical generation: a review", IET Generation, Transmission \& Distribution, 12(13), pp. 3107-3113 (2018).

39. Montgomery, D.C., Design and Analysis of Experiments, 8th Ed., John Wiley \& Sons, New Jersey, Hoboken, USA (2013).

40. Mirjalili, S., Mirjalili, S.M., and Lewis, A. "Grey wolf optimizer", Adv. Eng. Softw., 69, pp. 46-61 (2014).

41. Ileri, E., Karaoglan, A.D., and Akpinar, S. "Optimizing cetane improver concentration in biodiesel-diesel blend via grey wolf optimizer algorithm", Fuel, 273, Article no. 117784 (2020).

\section{Biographies}

Deniz Perin received MSc and PhD degrees in Physics from Balikesir University in 2008 and 2015, respectively. He studied Magnetic Flux Leakage (MFL) and Magnetic Non-destructive Testing in PhD program at Cardiff University Wolfson Centre. From 2017 till now, he has been working on magnetic design and simulation of synchronous generators with Ansys Maxwell at Isbir Electric Company Research \& Development Department as a research and development expert.

Aslan Deniz Karaoglan received a diploma degree in Industrial Engineering from Gazi University in 2001 (Turkey). Then, he received MSc from Balikesir Üniversity (Turkey) in 2006 and $\mathrm{PhD}$ in Industrial Engineering from Dokuz Eylul University in 2010 (Turkey). His research interests are design of experiments, statistical process control, artificial intelligence, nature-inspired algorithms, and optimization. He is an Associate Professor at Balikesir University (Turkey), Department of Industrial Engineering.

Kemal Yilmaz received a diploma in Mechatronics Engineering from Karabuk University (Turkey), Faculty of Technology in 2015. Since 2017, he has been working as a project and simulation engineer at Isbir Electric Company Research \& Development Department. 